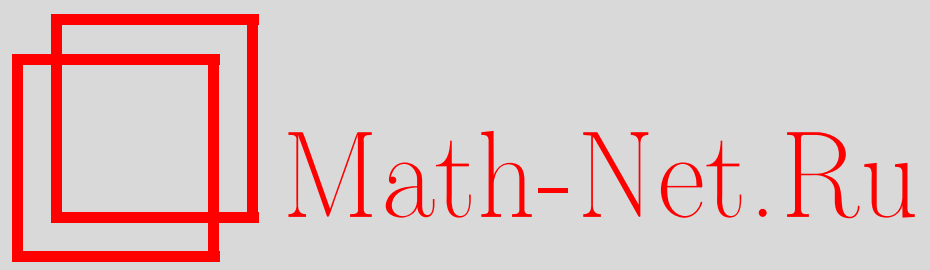

С. Грибок, Мудрецы, колпаки и арифметика конечных полей, Квант, 2019, номер 4, 5-13

DOI: https://doi.org/10.4213/kvant20190402

Использование Общероссийского математического портала Math-Net.Ru подразумевает, что вы прочитали и согласны с пользовательским соглашением http://www.mathnet.ru/rus/agreement

Параметры загрузки:

IP : 54.198 .55 .26

26 апреля 2023 г., 13:10:33

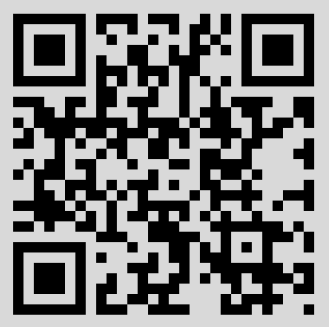




\section{Мудрецы, колпаки и арифметика конечных полей}

\section{С.ГРИБОК}

Испытание 1. Два из трех. О двоичных наборах, кубах и шарах

Давным-давно правил в Персии царь Шахрияр. Цельми днями иарь пировал, охотился и развлекался, устраивая жестокие испытания для своих придворных. $B$ то время как иарь проводил время в праздности, решением всех запутанных государственных дел и управлением страной занимался совет придворных мудречов.

Однаждь иарь решил проверить, так ли уж умны его придворные мудрецы. Он приказал позвать трех мудречов и объявил условия испытания, которое ожидает их завтра. Каждому мудреиу на голову наденут колпак белого или черного ивета. Каждый сможет видеть чужие колпаки, но не будет знать, какого ивета его колпак. Какой именно колпак надеть на каждого мудреца, решит палач, триждь подбросив монету, одна сторона которой окрашена в бельй ивет, а другая в черный. Затем каждому мудрецу дадут перо и лист пергамента, на котором он напишет, какого ивета его колпак. Если хотя бы двое мудрецов ответят правильно, всех наградят, если же хотя бы двое ошибутся, всех мудрецов казнят. Подавать друг другу какие-либо знаки во время испьтания запрещено. За ночь перед испытанием у мудрецов есть возможность договориться и выработать еди ную стратегию. Как следует действовать мудречам, чтобы максимизировать шансы остаться в живых?

Докажем, что не существует способа, позволяющего сделать вероятность выигрыша мудрецов больше чем 3/4 .

DOI: https://doi.org/10.4213/kvant20190402
Пусть испытание повторяется много раз. Пусть мудрецам удалось разработать метод, позволяющий двум мудрецам из трех угадывать цвета своих колпаков в среднем чаще, чем в трех играх из четырех. Таким образом, из 12 ответов мудрецов, прозвучавших в течение четырех игр, в среднем более 6 ответов будут верными $(6=3 \cdot 2)$. Но это невозможно, так как каждый мудрец угадывает цвет своего колпака в одном случае из двух, а значит, из 12 ответов в среднем ровно 6 ответов верны.

Всего есть 8 различных вариантов распределения колпаков между тремя мудрецами. Вероятность выигрыша $3 / 4$ означает, что мудрецы побеждают в шести вариантах, а проигрывают в двух вариантах из восьми.

Попробуем построить такую стратегию мудрецов, которая позволит им выигрывать в шести случаях из восьми.

Для удобства записи наборов колпаков будем использовать цифру 0 для обозначения белого колпака, а цифру 1 для обозначения черного колпака. Например, запись 001 будет означать, что первым двум мудрецам достались белые колпаки, а третий мудрец получил черный колпак.

Выберем два «смертельных» набора колпаков, которые мудрецы не смогут угадать. Например, пусть смертельными будут наборы 000 и 111. Мы хотим, чтобы на всех остальных наборах мудрецы смогли выиграть. Как этого добиться?

Прежде всего заметим, что для любого не смертельного набора колпаков один из мудрецов видит на соседях колпаки одного и того же цвета. Стратегия этого мудреца ясна: если видишь на соседях два колпака одного цвета, называй другой цвет. Оче- 
видно, для любого не смертельного набора эта стратегия работает и гарантированно позволит мудрецу угадать цвет своего колпака.

Займемся оставшимися двумя мудрецами, каждый из которых видит на соседях колпаки разных цветов. Чтобы выиграть, достаточно, чтобы хотя бы один из этих мудрецов назвал цвет своего колпака правильно. Заметим также, что их собственные колпаки одного и того же цвета. Значит, если один из мудрецов назовет белый цвет, а другой - черный, то один обязательно угадает. Но как мудрецы узнают, какой цвет называть, если они оба видят одну и ту же картину - на соседях колпаки разных цветов? Дело в том, что если присмотреться внимательнее, мы заметим: в том, что видят эти два мудреца, есть некоторые различия. Пусть, например, мудрецы встали в круг. Тогда один из двух мудрецов видит слева белый колпак, а справа - черный. Другой мудрец видит иную картину: слева черный колпак, а справа - белый.

Теперь легко придумать правило, которое позволит одному из этих мудрецов угадать цвет своего колпака: если видишь на соседях колпаки разных иветов, называй тот ивет, который видишь справa (заметим, что также годится и «зеркально-симметричное» правило: если видишь на соседях колпаки разных цветов, то называй тот цвет, который видишь слева).

Итак, задача решена, но не будем торопиться. Во-первых, подумаем, нельзя ли упростить правила, которым должны следовать мудрецы. Нетрудно догадаться, что два найденных нами правила можно переформулировать в виде одного простого правила: называй НЕ тот цвет, который видишь на соседе слева.

Во-вторых, построим геометрическую интерпретацию задачи о трех колпаках. Удобно представить множество вариантов распределения колпаков в виде куба (рис.1). Каждый набор колпаков соответствует одной из вершин куба. Если два набора колпаков отличаются лишь цветом одного колпака (такие наборы называются

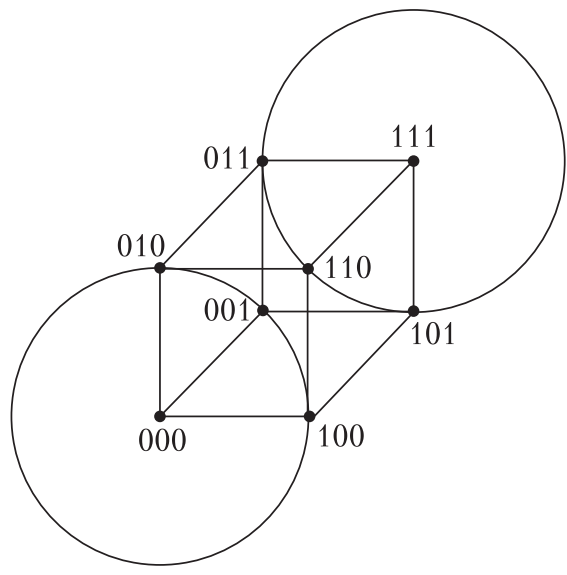

Pис. 1

соседними), они расположены в вершинах, соединенных ребром.

Достроим теперь два шара, центры которых находятся в смертельных точках 000 и 111, а радиусы равны ребру куба. Каждый шар покрывает четыре точки куба. Каждая точка куба либо находится на поверхности, либо в центре одного из шаров. В таких случаях говорят, что построено «покрытие куба непересекающимися шарами». Если точка оказалась в центре шара, мудрецы не угадают цвета своих колпаков, если же точка лежит на поверхности шара, большинство мудрецов назовут свои цвета правильно.

\section{Испытание 2. Четыре из семи. О многочленах и кодовых множествах}

На следуюший день к царю привели семерых мудречов. Правитель объявил, ито сегодня состоится еще одно испьтание.

Правила те же, что и вчера: каждому мудрецу на голову наденут колпак белого либо черного ивета, после чего каждый мудреи должен записать, какого ивета по его мнению колпак на его голове. Если хотя бы четверо мудрецов ответят правильно, всех наградят, если же хотя бы четверо ошибутся, всех казнят.

Как следует действовать мудрецам, чтобы и на этот раз свести вероятность казни к минимуму?

Опять начнем с нижней оценки вероятности казни. Докажем, что не существует 
способа, позволяющего сделать вероятность выигрыша больше чем 7/8. Доказательство такое же, как и в первой задаче, с точностью до замены чисел.

Пусть мудрецам удалось разработать метод, позволяющий четырем мудрецам из семи угадывать цвета своих колпаков в среднем чаще, чем в семи играх из восьми. Таким образом, из 56 ответов мудрецов, прозвучавших в течение восьми игр, в среднем более 28 ответов будут верными $(28=7 \cdot 4)$. Но это невозможно, так как каждый мудрец угадывает цвет своего колпака в одном случае из двух, а значит, из 56 ответов в среднем ровно 28 ответов верны.

Всего есть 128 различных вариантов распределения колпаков между семью мудрецами. Вероятность выигрыша $7 / 8$ означает, что мудрецы побеждают в 112 вариантах, а проигрывают в 16 вариантах из 128.

По аналогии с первой задачей теперь надо как-то выбрать 16 смертельных наборов. Но как это сделать?

В предыдущей задаче все наборы из трех колпаков мы расставили по вершинам куба. Теперь колпаков семь, а значит, множество наборов соответствует вершинам семимерного гиперкуба. Представить эту фигуру сложно, однако это не мешает нам порассуждать о ее свойствах.

Заметим, что так как мудрецов семеро, то для каждого набора колпаков существует семь соседних наборов, следовательно, каждый 7-мерный шар единичного радиуса с центром в вершине $N$-мерного куба покрывает ровно 8 точек куба. Поскольку $128=16 \cdot 8$, то теоретически для покрытия всех 128 вершин 7-мерного куба как раз должно хватить шестнадцати непересекающихся 7-мерных шаров, в центре каждого из которых мы поместим смертельный набор. Вот только как это сделать на практике?

Будем называть расстоянием между двумя наборами число позиций, в которых эти наборы отличаются. Расстояние между соседними наборами равно единице. Теперь сформулируем свойства множества смертельных наборов, которое мы хотим построить.
1) Всего в множестве 16 наборов.

2) Расстояние между любыми двумя наборами не меньше трех (чтобы шары, в центрах которых находятся смертельные наборы, не пересекались).

3) Циклический сдвиг смертельного набора также является смертельным набором (иначе разным мудрецам придется использовать различные стратегии).

Интуитивно ясно, что смертельные наборы должны быть равномерно распределены на множестве всех возможных наборов.

Можно попробовать представить каждый набор колпаков как двоичную запись некоторого целого числа от 0 до 127 , а множество смертельных наборов - как элементы некоторой арифметической прогрессии, однако традиционные арифметические операции плохо подходят для обеспечения нужных нам свойств 2 и 3.

Так как традиционная арифметика нам не подходит, воспользуемся «нетрадиционной» арифметикой, а именно, так называемой арифметикой многочленов над полем $\{0,1\}$.

Прежде всего определим операцию сложения двух наборов колпаков. Сумма двух наборов длины $n$ - это набор длины $n$, каждый элемент которого равен сумме по модулю 2 соответствующих элементов исходных наборов.

Напомним правила сложения по модулю 2 :

$$
\begin{aligned}
& 0+0=0, \\
& 0+1=1, \\
& 1+0=1, \\
& 1+1=0 .
\end{aligned}
$$

Пример:

$$
00110101+11110000=11000101 .
$$

Чтобы определить операции умножения и деления наборов колпаков, удобно записывать наборы в виде многочленов. Набор колпаков $v_{0}, v_{1}, v_{2}, \ldots, v_{n-1}$ можно записать в виде многочлена от переменной $x$ : $v_{0}+v_{1} \cdot x+v_{2} \cdot x^{2}+\ldots+v_{n-1} \cdot x^{n-1}$. Таким образом, множество наборов длины $n$ эквивалентно множеству многочленов, степени которых не превышают $n-1$.

Теперь определим произведение двух наборов как набор, соответствующий мно- 
гочлену, полученному при обычном умножении многочленов исходных двух наборов.

$$
\text { Пример: }
$$

$101 \cdot 1111=$

$$
\begin{gathered}
=\left(1+0 \cdot x+1 \cdot x^{2}\right) \cdot\left(1+1 \cdot x+1 \cdot x^{2}+1 \cdot x^{3}\right)= \\
=\left(1+x^{2}\right) \cdot\left(1+x+x^{2}+x^{3}\right)= \\
=1+x+x^{2}+x^{3}+x^{2}+x^{3}+x^{4}+x^{5}= \\
=1+x+(1+1) \cdot x^{2}+(1+1) \cdot x^{3}+x^{4}+x^{5}= \\
=1+x+0 \cdot x^{2}+0 \cdot x^{3}+x^{4}+x^{5}=110011 .
\end{gathered}
$$

Аналогично, деление двух наборов определим как деление соответствующих многочленов. Деление многочленов нетрудно производить уголком, подобно делению обычных чисел, но без переноса разрядов.

Пример приведен на рисунке 2.

В дальнейшем будем использовать термины «набор длины $n$ и «многочлен, степень которого не превышает $n-1 »$ как синонимы.

Построим множество смертельных наборов как множество многочленов, степень которых не превышает $n-1$ и которые делятся без остатка на некоторый специальный многочлен $g(x)$.

Кодовое множество размерности $n$, порожденное многочленом $g(x)$, - это множество многочленов, степень которых не превышает $n-1$, делящихся без остатка на $g(x)$

Элементы кодового множества будем называть кодовыми наборами.

Лемма. Число наборов в кодовом множестве размерности $n$, порожденном многочленом степени $k-1$, равно $2^{n-k+1}$

Доказательство. Порожденные наборы получаются при умножении набора длины $k$ на все возможные наборы длины $n-k+1$, число которых в точности равно $2^{n-k+1}$

$$
\begin{aligned}
& x^{5}+x^{4}+0 \cdot x^{3}+0 \cdot x^{2}+x+1 \mid x^{2}+0 \cdot x+1 \\
& x^{5}+0 \cdot x^{4}+x^{3} \quad \overline{x^{3}+x^{2}+x+1} \\
& x^{4}+x^{3}+0 \cdot x^{2} \\
& \frac{x^{4}+0 \cdot x^{3}+x^{2}}{x^{3}+x^{2}+x} \\
& \frac{x^{3}+0 \cdot x^{2}+x}{x^{2}+0 \cdot x+1} \\
& \frac{x^{2}+0 \cdot x+1}{0}
\end{aligned}
$$

В данном случае мы хотим получить кодовые наборы длины 7 , а число элементов в кодовом множестве должно быть равно 16. Поэтому по лемме надо использовать порождающий набор длины 4, или, другими словами, многочлен степени 3.

Возьмем такой многочлен:

$$
g(x)=1+x+x^{3} .
$$

Множество кодовых наборов, порожденных этим многочленом, приведено в таблице.

\begin{tabular}{|l|l|}
\hline 0000000 & $0 \cdot g(x)=0$ \\
\hline 1101000 & $1 \cdot g(x)=1+x+x^{2}$ \\
\hline 0110100 & $x \cdot g(x)=x+x^{2}+x^{4}$ \\
\hline 1011100 & $(1+x) \cdot g(x)=1+x^{2}+x^{3}+x^{4}$ \\
\hline 0011010 & $x^{2} \cdot g(x)=x^{2}+x^{3}+x^{5}$ \\
\hline 1110010 & $\left(1+x^{2}\right) \cdot g(x)=1+x+x^{2}+x^{3}+x^{5}$ \\
\hline 0101110 & $\left(x+x^{2}\right) \cdot g(x)=x+x^{3}+x^{4}+x^{5}$ \\
\hline 1000110 & $\left(1+x+x^{2}\right) \cdot g(x)=1+x^{4}+x^{5}$ \\
\hline 0001101 & $x^{3} \cdot g(x)=x^{3}+x^{4}+x^{6}$ \\
\hline 1100101 & $\left(1+x^{3}\right) \cdot g(x)=1+x+x^{4}+x^{6}$ \\
\hline 0111001 & $\left(x+x^{3}\right) \cdot g(x)=x+x^{2}+x^{3}+x^{6}$ \\
\hline 1010001 & $\left(1+x+x^{3}\right) \cdot g(x)=1+x^{2}+x^{6}$ \\
\hline 0010111 & $\left(x^{2}+x^{3}\right) \cdot g(x)=x^{2}+x^{4}+x^{5}+x^{6}$ \\
\hline 1111111 & $\left(1+x^{2}+x^{3}\right) \cdot g(x)=1+x+x^{2}+x^{3}+x^{4}+x^{5}+x^{6}$ \\
\hline 0100011 & $\left(x+x^{2}+x^{3}\right) \cdot g(x)=x+x^{5}+x^{6}$ \\
\hline 1001011 & $\left(1+x+x^{2}+x^{3}\right) \cdot g(x)=1+x^{3}+x^{5}+x^{6}$ \\
\hline
\end{tabular}


Свойство 1 выполнено: множество содержит 16 наборов. Легко проверить, что свойство 3 также выполнено: для каждого набора из множества циклический сдвиг этого набора также принадлежит этому множеству. Осталось доказать свойство 2. Для этого сначала докажем следующее утверждение.

Теорема 1. Если наборы т и и являются кодовыми наборами, то набор $ш=v+u$ также является кодовым набором.

Доказательство. Имеем

$$
\begin{aligned}
v(x)+u(x)=g(x) & \cdot a(x)+g(x) \cdot b(x)= \\
& =g(x) \cdot(a(x)+b(x)) .
\end{aligned}
$$

Если бы свойство 2 не выполнялось, то нашлись бы два набора на расстоянии 1 или 2 , а значит, по теореме 1 , существовал бы набор, равный сумме этих наборов, в котором было бы всего одна или две единицы. Однако ни один из наборов, перечисленных выше, не содержит ровно одну или ровно две единицы. Таким образом, свойство 2 также выполнено.

Итак, мы построили покрытие семимерного куба непересекающимися шарами.

Можно сказать, задача мудрецов заключается в том, чтобы найти вершину гиперкуба, которая соответствует набору их колпаков. Ни один из мудрецов не знает цвет своего колпака, однако он видит цвета всех остальных колпаков, т.е. этому мудрецу известны все цифры в наборе, кроме одной - той, которая соответствует его собственной позиции. Таким образом, с точки зрения этого мудреца набор колпаков соответствует одной из двух вершин гиперкуба, соединенных ребром. Задача мудреца - выбрать правильную вершину этого ребра. Для простоты терминологии будем говорить, что мудрец «находится на ребре» гиперкуба.

Определим теперь стратегии мудрецов. Прежде всего, если мудрец находится на ребре, одна из вершин которого является кодовым набором, то мудрец должен назвать цвет, соответствующий другой вершине. Это правило гарантирует, что если набор колпаков является смертельным набором, то все мудрецы ошибутся, если же набор колпаков не является смертель- ным набором, то по крайней мере один из мудрецов назовет цвет своего колпака правильно,

Пусть теперь мудрец находится на ребре, ни одна из вершин которого не является кодовым набором. Каждая из этих вершин покрыта некоторым шаром, т.е. можно сказать, что мудрец находится на ребре, соединяющем два шара. Центры этих шаров находятся на расстоянии 3 друг от друга. Значит, соответствующие наборы колпаков различаются в трех позициях: $i$, $j, k$. Одна из этих позиций - это позиция самого мудреца. Для определенности, пусть это будет позиция $i$.

Мудрец смотрит, какая из позиций, $j$ или $k$, встретится первой при обходе семерки мудрецов по часовой стрелке, начиная с позиции $i$, и называет цвет, соответствующий этому шару.

Возьмем произвольную точку $X$ гиперкуба, не лежащую в центре шара, и докажем, что большинство мудрецов, оказавшихся в точке $X$, правильно назовут свой цвет.

Точка $X$ принадлежит некоторому шару $S$. Мудрец $W_{1}$, находящийся на ребре, ведущем в центр шара $S$, очевидно, назовет свой цвет правильно. Остальные мудрецы сидят на ребрах между шаром $S$ и другими шарами, центры которых находятся на расстоянии 2 от точки $X$. Рассмотрим один из этих шаров, $S_{1}$. Расстояние от центра $S_{1}$ до $X$ равно двум, а значит, есть два ребра, ведущих из $X$ в $S_{1}$. На этих ребрах сидят два мудреца, $W_{2}$ и $W_{3}$. Нетрудно убедиться в том, что либо $W_{2}$, либо $W_{3}$ угадает свой цвет. Действительно, пусть, например, при обходе семерки мудрецов по часовой стрелке сначала встречается $W_{1}$, затем $W_{2}$ и наконец $W_{3}$. В этом случае мудрец $W_{3}$ назовет свой цвет правильно, так как при движении по часовой стрелке ему первой встретится позиция $W_{1}$, и он назовет свой цвет, правильно предполагая, что находится в шаре $S$.

Аналогично, все оставшиеся мудрецы разобьются на пары, в каждой из которых один из мудрецов угадает свой цвет.

Итак, решение найдено. Как его упростить? 
Как мы помним, оптимальная стратегия в первом испытании заключалась в том, что мудрецы вставали в круг и каждый мудрец называл цвет, не совпадающий с цветом колпака его соседа слева. Попробуем обобщить эту стратегию. Будем искать решение следующего вида.

Пусть выбрано некоторое число $k, 0<$ $<k<n$, и $k$ различных чисел $0<i_{1}<\ldots$ $\ldots<i_{k}<n$. Мудрецы становятся в круг, и каждый мудрец подсчитывает число черных колпаков у $k$ мудрецов на расстояниях $i_{1}, \ldots, i_{k}$ справа от него. Если это число четно, мудрец называет черный цвет, иначе он называет белый цвет.

Будем называть такую стратегию мудрецов чиклической стратегией.

Сопоставим циклической стратегии набор $h$ длины $n$, в котором в позициях $0, i_{1}, \ldots, i_{k}$ стоят единицы, а в остальных позициях стоят нули. Этот набор можно записать и в виде многочлена: $h(x)=1+$ $+x^{\dot{i}_{1}}+\ldots+x^{i_{k}}$.

Пусть цвета колпаков, доставшихся мудрецам, заданы набором $v=\left(v_{0}, v_{1}, v_{2}, \ldots\right.$ $\left.\ldots, v_{n-1}\right)$. Пусть мудрецы встали в круг в порядке номеров по часовой стрелке. Какой цвет должен назвать мудрец с индексом $i$, если он использует циклическую стратегию $h=\left(h_{0}, h_{1}, h_{2}, \ldots, h_{n-1}\right)$ ?

По определению циклической стратегии, $i$-й мудрец должен подсчитать сумму элементов в наборе $v$, соответствующих единицам в наборе $h$ при обходе набора $v$ против часовой стрелки:

$$
v_{i-1} \cdot h_{1}+v_{i-2} \cdot h_{2}+\ldots+v_{i+1} \cdot h_{n-1} .
$$

Если эта сумма равна нулю, мудрец называет единицу (черный цвет), в противном случае он называет ноль (белый цвет). Таким образом, цвет, названный $i$-м мудрецом, можно записать как

$$
v_{i-1} \cdot h_{1}+v_{i-2} \cdot h_{2}+\ldots+v_{i+1} \cdot h_{n-1}+1 .
$$

Справедлива следующая удивительная теорема.

Теорема 2. Пусть $h(x)-$ многочлен, соответствующий некоторой чиклической стратегии мудрещов, а v $(x)$ - многочлен, соответствуюший некоторому набору колпаков. Тогда многочлен $v(x) \cdot h(x)\left(\bmod \left(x^{n}+1\right)\right)$ задает позиции мудрецов, угадавших ивета своих колпаков.

Доказательство. Запишем:

$$
\begin{aligned}
& v(x) \cdot h(x)\left(\bmod \left(x^{n}+1\right)\right)= \\
& =\left(v_{0}+v_{1} \cdot h_{n-1}+\ldots+v_{n-1} \cdot h_{1}\right)+ \\
& +\left(v_{1}+v_{2} \cdot h_{n-1}+\ldots+v_{0} \cdot h_{1}\right) \cdot x+\ldots \\
& \quad \ldots+\left(v_{n-1}+v_{0} \cdot h_{n-1}+\ldots+v_{n-2} \cdot h_{1}\right) \cdot x^{n-1} .
\end{aligned}
$$

Цвет, который назовет первый мудрец, задается выражением $v_{1} \cdot h_{n-1}+\ldots+v_{n-1} \cdot h_{1}+$ +1 . Таким образом, первый мудрец угадает свой цвет, если $v_{0}=v_{1} \cdot h_{n-1}+\ldots$ $\ldots+v_{n-1} \cdot h_{1}+1$, или, что то же самое, если

$$
v_{0}+v_{1} \cdot h_{n-1}+\ldots+v_{n-1} \cdot h_{1}=1 .
$$

Аналогично можно убедиться в истинности теоремы для других мудрецов.

Заметим, что выбранный нами кодовый многочлен $g(x)=1+x+x^{3}$ делит без остатка $x^{7}+1$. Что произойдет, если мудрецы используют циклическую стратегию, где

$$
\begin{array}{r}
h(x)= \\
=\left(x^{7}+1\right) / g(x)=\left(x^{7}+1\right) /\left(1+x+x^{3}\right)= \\
=1+x+x^{2}+x^{4} ?
\end{array}
$$

Если набор колпаков $v(x)$ принадлежит кодовому множеству, то

$$
\begin{aligned}
v(x) & \cdot h(x)\left(\bmod \left(x^{n}+1\right)\right)= \\
& =a(x) \cdot g(x) \cdot h(x)\left(\bmod \left(x^{n}+1\right)\right)=0,
\end{aligned}
$$

таким образом, никто из мудрецов не угадает цвет своего колпака.

Если $v(x)$ не принадлежит кодовому множеству, то, как мы установили выше, $v(x)$ находится на расстоянии 1 от некоторого кодового слова $u(x)$. Тогда

$$
\begin{gathered}
\left(u(x)+x^{k}\right) \cdot h(x)\left(\bmod \left(x^{n}+1\right)\right)= \\
=(a(x) \cdot g(x) \cdot h(x)+ \\
\left.+x^{k} \cdot h(x)\right)\left(\bmod \left(x^{n}+1\right)\right)= \\
=\left(x^{k} \cdot h(x)\right)\left(\bmod \left(x^{n}+1\right)\right) .
\end{gathered}
$$

Это циклический сдвиг многочлена $h(x)$. Следовательно, по теореме 2 число мудрецов, которые угадают цвета своих колпа- 
ков, равно числу единиц в многочлене $h(x)$, т.е. равно 4. Таким образом, для любого набора колпаков, не принадлежащего кодовому множеству, большинство мудрецов угадает цвета своих колпаков.

Словесная формулировка этой стратегии: если число черных колпаков у мудрецов, стояших на расстояниях 1, 2, 4 от меня по часовой стрелке, четно, то следует назвать черныйчвет, иначе-бельй.

\section{Испытание 3. Восемь из пятнадцати.} О свойствах порождающего многочлена

На третий день царь приказал привести к нему 15 мудрецов и объявил, что сегодня состоится последнее испытание.

Как и в предьдущие дни, каждому мудречу на голову наденут колпак белого либо черного цвета, после чего каждьй мудрецдолжен записать, какого цвета по его мнению колпак на его голове. Если хотя бы восемь мудрецов ответят правильно, всех наградят, если же хотя бь восемь ошибутся, всех казнят.

Как следует действовать мудрецам, чтобь свести вероятность казни к минимyмy?

Опять начнем с нижней оценки вероятности казни. Докажем, что не существует способа, позволяющего сделать вероятность выигрыша больше чем 15/16 . Доказательство аналогично предыдущим.

Пусть мудрецам удалось разработать метод, позволяющий восьми мудрецам из пятнадцати угадывать цвета своих колпаков в среднем чаще, чем в пятнадцати играх из шестнадцати. Таким образом, из 240 ответов мудрецов, прозвучавших в течение шестнадцати игр, в среднем более 120 ответов будут верными $(120=15 \cdot 8)$. Но это невозможно, так как каждый мудрец угадывает цвет своего колпака в одном случае из двух, а значит, из 240 ответов в среднем ровно 120 ответов верны.

Всего существует $2^{15}$ различных наборов колпаков. Заметим, что 15-мерный шар покрывает 16 точек 15-мерного куба, таким образом, для покрытия всех $2^{15}$ точек куба потребуется $2^{15} / 16=2048$ шаров. Итак, кодовое множество включает 2048 наборов. Выписывать все эти наборы было бы слишком долго. Докажем три теоремы, которые помогут нам избавиться от выписывания 2048 наборов.

Теорема 3.1. Пусть $g(x)$ делит $x^{n}+1$. Тогда чиклический сдвиг любого кодового набора также является кодовым набором.

Доказательство. Пусть $v=\left(v_{0}, v_{1}, \ldots\right.$ $\left.\ldots, v_{n-2}, v_{n-1}\right)-$ кодовый набор. Обозначим через $v^{\prime}$ набор, полученный циклическим сдвигом $v$ на одну позицию вправо:

$$
\begin{gathered}
v^{\prime}=\left(v_{n-1}, v_{0}, v_{1}, \ldots, v_{n-3}, v_{n-2}\right)= \\
=v_{n-1}+v_{0} \cdot x+v_{1} \cdot x^{2}+\ldots \\
\ldots+v_{n-3} \cdot x^{n-2} \cdot x^{n-1}= \\
=v_{n-1}+x \cdot v(x)+v_{n-1} \cdot x^{n}= \\
=x \cdot v(x)+v_{n-1} \cdot\left(x^{n}+1\right)= \\
=x \cdot a(x) \cdot g(x)+v_{n-1} \cdot b(x) \cdot g(x)= \\
=\left(x \cdot a(x)+v_{n-1} \cdot b(x)\right) \cdot g(x) .
\end{gathered}
$$

Теорема 3.2. Пусть $g(x)$ делит $x^{n}+1$ и не делит ни один из многочленов $x^{k}+1$, где $k<n$. Тогда расстояние между любьми кодовыми наборами не меньше чем 3.

Доказательство. Пусть существуют два кодовых набора $u$ и $v$, расстояние между которыми меньше трех. Рассмотрим сумму этих наборов $w=u+v$. Так как $u$ и $v$ отличаются не более чем в двух позициях, в $ш$ не более двух единиц. По теореме 1 w $(x)$ является кодовым набором, а по теореме 3.1 любой циклический сдвиг ш также является кодовым набором. Рассмотрим кодовый набор $s-$ циклический сдвиг ж, где единица стоит в позиции 0. Если в $s$ всего одна единица, то, очевидно, $s=1$ не может быть кодовым словом. Если же в $s$ две единицы, то $s=x^{k}+1$ для некоторого $k<n$. Однако, по условию, $g(x)$ не делит ни один из многочленов $x^{k}+1$. Пришли к противоречию.

Теорема 3.3. Пусть $g(x)$ делит $x^{n}+1$. Пусть стратегия мудрецов определяется многочленом $h(x)=\left(x^{n}+1\right) / g(x)$. Тогда:

1) для любого набора колпаков из кодового множества ни один из мудречов не угадает ивет своего колпака;

2) для любого набора колпаков на расстоянии 1 от кодового множества число мудрецов, угадавших ивет своих колпа- 
ков, равно числу единии в многочлене $h(x)$.

Доказательство. Если набор колпаков $v(x)$ принадлежит кодовому множеству, Tо

$$
\begin{aligned}
& v(x) \cdot h(x)\left(\bmod \left(x^{n}+1\right)\right)= \\
& =a(x) \cdot g(x) \cdot h(x)\left(\bmod \left(x^{n}+1\right)\right)= \\
& \quad=a(x) \cdot\left(x^{n}+1\right)\left(\bmod \left(x^{n}+1\right)\right)=0,
\end{aligned}
$$

таким образом, никто из мудрецов не угадает цвет своего колпака.

Если $v(x)$ находится на расстоянии 1 от некоторого кодового слова $u(x)$, то

$$
\begin{gathered}
\left(u(x)+x^{k}\right) \cdot h(x)\left(\bmod \left(x^{n}+1\right)\right)= \\
=(a(x) \cdot g(x) \cdot h(x)+ \\
\left.+x^{k} \cdot h(x)\right)\left(\bmod \left(x^{n}+1\right)\right)= \\
=\left(x^{k} \cdot h(x)\right)\left(\bmod \left(x^{n}+1\right)\right) .
\end{gathered}
$$

Это циклический сдвиг многочлена $h(x)$. Следовательно, по теореме 2 число мудрецов, которые угадают цвета своих колпаков, равно числу единиц в многочлене $h(x)$.

Теперь можно приступить к построению оптимальной стратегии для 15 мудрецов.

Из теорем 3.1 и 3.2 следует, что для генерации кодового множества нужен многочлен, который делит $x^{15}+1$. Разложим многочлен $x^{15}+1$ на множители:

$$
\begin{aligned}
x^{15}+1= & \\
=(1+ & \left.x+x^{2}+x^{3}+x^{4}\right) \cdot\left(1+x^{3}+x^{4}\right) \times \\
& \times\left(1+x+x^{4}\right) \cdot\left(1+x+x^{2}\right) \cdot(1+x) .
\end{aligned}
$$

В кодовом множестве, которое мы хотим построить, должно быть 2048 элементов, поэтому по лемме для генерации кодового множества нужен многочлен четвертой степени.

В разложении $x^{15}+1$ три многочлена четвертой степени:

1) $1+x+x^{2}+x^{3}+x^{4}$;

2) $1+x+x^{4}$

3) $1+x^{3}+x^{4}$.

Многочлен $1+x+x^{2}+x^{3}+x^{4}$ нам не подходит, так как он делит $x^{5}+1$, а значит, не выполняются условия теоремы 3.2. Но зато для любого из оставшихся двух мно- гочленов условия теоремы 3.2 выполнены (оставляем проверку этого в качестве задания читателю).

Выберем для генерации кодового множества многочлен $1+x+x^{4}$. В соответствии с теоремой 3.3 выберем стратегию мудрецов, соответствующую многочлену $\left(x^{n}+1\right) / g(x)$. Имеем:

$$
\begin{aligned}
& \left(x^{15}+1\right) /\left(x^{4}+x+1\right)= \\
& \quad=1+x+x^{2}+x^{3}+x^{5}+x^{7}+x^{8}+x^{11} .
\end{aligned}
$$

В этом многочлене 8 единиц, следовательно, по теореме 3.3 для каждого набора колпаков, не входящего в кодовое множество, 8 мудрецов из 15 угадают цвета своих колпаков.

Итак, стратегия, позволяющая 15 мудрецам выиграть с вероятностью 15/16, определяется многочленом $1+x+x^{2}+$ $+x^{3}+x^{5}+x^{7}+x^{8}+x^{11}$. Словесная формулировка этой стратегии: если число черных колпаков у мудрещов, стоящих на расстояниях 1, 2, 3, 5, 7, 8, 11 от меня по часовой стрелке, четно, то следует назвать черный ивет, иначе - белый.

\section{Заключение. О помехоустойчивом кодировании и кодах Хэмминга}

Возможно, вдумчивый читатель уже давно задается вопросом: являются ли конечные поля, кодовые множества и порождающие многочлены абстрактной теорией, которая использовалась лишь мудрецами царя Шахрияра, или же эти понятия имеют и иное применение? Пришло время ответить на этот вопрос. Теория, которая помогла мудрецам, повсеместно используется в современных системах хранения и передачи информации. Одно из важнейших приложений этой теории - это помехоустойчивое кодирование. Проиллюстрируем задачу помехоустойчивого кодирования на простом примере.

Пусть мудрец Азат, живущий в пункте А, должен передать сообщение, состоящее из нулей и единиц, мудрецу Бахиру, живущему в пункте Б. Известно, что в процессе передачи один из символов сообщения может быть искажен помехами, т.е. один из нулей может превратиться в единицу 
либо одна из единиц может стать нулем. Существует ли способ закодировать сообщение таким образом, чтобы, даже несмотря на воздействие помех, исходное сообщение могло бы быть безошибочно восстановлено в пункте Б?

Конечно, Азат может просто трижды продублировать сообщение. Если одна из копий окажется испорченной помехами, то сообщение можно восстановить по двум другим копиям. Однако передача трех копий сообщения может быть слишком долгой и дорогой. Кроме того, может оказаться, что в столь длинной передаче помехи могут повлиять более чем на один символ. Есть ли более эффективный способ кодирования?

Оказывается, такой способ существует, и нам уже известна вся теория, необходимая для его реализации. Этот метод называется кодированием Хэмминга в честь его изобретателя, американского математика Ричарда Хэмминга.

Азат и Бахир выбирают многочлен $g(x)$ (порождающий многочлен), удовлетворяющий условиям теоремы 3.2, и вместо исходного сообщения $a(x)$ Азат передает из пункта А в пункт Б сообщение $u(x)=a(x) \cdot g(x)$. Теперь Бахир должен декодировать полученное сообщение $v(x)$, один из символов которого, возможно, искажен.

Для начала Бахир вычисляет синдром полученного сообщения

$$
s(x)=v(x) \cdot h(x)\left(\bmod \left(x^{n}+1\right)\right) .
$$

Если сообщение не было изменено помехами, то

$$
\begin{aligned}
& s(x)=v(x) \cdot h(x)\left(\bmod \left(x^{n}+1\right)\right)= \\
& =a(x) \cdot g(x) \cdot h(x)\left(\bmod \left(x^{n}+1\right)\right)= \\
& =a(x) \cdot\left(x^{n}+1\right)\left(\bmod \left(x^{n}+1\right)\right)=0 .
\end{aligned}
$$

Предположим теперь, что в переданном сообщении символ с индексом $k$ был изменен. Тогда

$$
\begin{gathered}
s(x)=\left(u(x)+x^{k}\right) \cdot h(x)\left(\bmod \left(x^{n}+1\right)\right)= \\
=(a(x) \cdot g(x) \cdot h(x)+ \\
\left.+x^{k} \cdot h(x)\right)\left(\bmod \left(x^{n}+1\right)\right)= \\
=\left(x^{k} \cdot h(x)\right)\left(\bmod \left(x^{n}+1\right)\right) .
\end{gathered}
$$

Это циклический сдвиг многочлена $h(x)$ на $k$ позиций.

Если синдром равен нулю, то Бахир знает, что переданное сообщение не было искажено, а значит, можно восстановить исходное сообщение по формуле

$$
a(x)=v(x) / g(x) .
$$

Если же синдром представляет собой циклический сдвиг $h(x)$ на $k$ позиций, то Бахир знает, что в переданном сообщении был испорчен символ с индексом $k$, a значит, исходное сообщение восстанавливается по формуле

$$
a(x)=\left(v(x)+x^{k}\right) / g(x) .
$$

\section{Численные значения: зачем и почему они нужны}

(Начало см. на с. 2)

разного рода жуликов и от недобросовестной рекламы. Любим же мы численные оценки, как и вообще знание, именно потому, что знание помогает выживанию и при правильном его применении улучшает жизнь. Это все хорошо, а что делать сейчас? Ответ прост - взять учебник физики (за все классы) и, медленно листая его, про каждую встреченную величину поду- мать, какие ее значения встречаются в жизни, какие могут встретиться в задачах. Интернет поможет вам найти ответы, а глядишь, и статью для «Кванта» напишите. А еще полезно посмотреть статьи А.А.Лукьянова про численные оценки - в интернете спросите «Лукьянов» и «в числах».

И последнее. Все ответы, приведенные в этой статье, реальные. Диалог в эпиграфе - тоже. Правда, тут же выяснилось, что собеседник шутил. А вот школьники, когда писали все эти ужасы, отнюдь не шутили. 